\title{
Terapia fotodinâmica associada a laser no tratamento endodôntico
}

Laser-associated photodynamic therapy in endodontic treatment

Terapia fotodinámica asociada con láser en el tratamiento endodóntico

Renally França de OLIVEIRA ${ }^{1}$

Liandra Pamela de Lima SILVA ${ }^{2}$

Fábio Victor Dias SILVA ${ }^{2}$

Kauana da Silva ANDRADE ${ }^{2}$

Thaynara Cavalcante Moreira ROMÃO ${ }^{2}$

Manuela Gouvêa Campêlo dos SANTOS ${ }^{3}$

Rachel Reinaldo ARNAUD ${ }^{4}$

${ }^{1}$ Cirurgiã-Dentista, Centro Universitário de João Pessoa, UNIPÊ, 58053-000 João Pessoa - PB, Brasil

${ }^{2}$ Graduação em Odontologia, Centro Universitário de João Pessoa, UNIPÊ, 58053-000 João Pessoa - PB, Brasil

${ }^{3}$ Doutora em Clínicas Odontológicas, Professora Adjunta do Departamento de Odontologia do UNIESP Centro Universitário, 58109-303 João Pessoa - PB, Brasil

${ }^{4}$ Professora Mestre, Departamento de Odontologia, Centro Universitário de João Pessoa, UNIPÊ, 58053-000 João Pessoa-PB, Brasil

\section{Resumo}

Introdução: A terapia fotodinâmica associada a laser tem sido estudada e aplicada em diversas especialidades da Odontologia objetivando o uso de técnicas menos invasivas. Objetivo: Este estudo tem como objetivo apresentar aos cirurgiões-dentistas e à sociedade a nova face da Odontologia voltada à utilização da terapia fotodinâmica associada a laser na prática endodôntica, bem como, apresentar os aspectos gerais da laserterapia. Material e Metódos: Trata-se de uma revisão de literatura, caracterizada como descritiva e exploratória. Foram realizadas buscas em livros, artigos científicos, monografias, dissertações e teses, no período de agosto de 2018 a maio de 2019. Esses dados foram obtidos por meio de bases de dados MedLine, PubMed, Scielo, LILACS e BBO. Resultados: Os resultados mostraram que a terapia fotodinâmica associada a laser é efetiva como coadjuvante ao tratamento endodôntico convencional, sendo uma das principais utilizações auxiliar na desinfecção dos canais radiculares, que consiste em utilizar uma fonte de luz com comprimento de onda específico capaz de estimular um agente fotossensível, que resulta em danos oxidativos, causando a morte celular. São observados também estudos do seu desempenho no preparo cavitário, controle da vitalidade pulpar, capeamento pulpar e pulpotomia, limpeza dos condutos radiculares, obturação e adesão dos cimentos endodônticos, retratamento, cirurgias periapicais, redução da dor e reparação pós-operatória. Conclusão: Concluiu-se que a terapia fotodinâmica utilizada como coadjuvante à terapia endodôntica convencional aumenta a previsibilidade de sucesso do tratamento, desde que, o conhecimento sobre os fundamentos e interações com os tecidos biológicos sejam esclarecidos e dominados pelo profissional.

Descritores: Endodontia; Lasers; Fotoquimioterapia; Terapia a Laser.

\section{Abstract}

Introduction: The photodynamic therapy associated with laser has been studied and applied in several Dentistry specialties, aiming the use of less invasive techniques. Objective: Therefore, the objective of this study was to present to dentists and society the new face of dentistry which use photodynamic therapy associated with laser in endodontic practice, as well as to present the general aspects of laser therapy. Material and method: It is a literature review, characterized as descriptive and exploratory. Searches were conducted in books, scientific articles, monographs, dissertations and theses, from August 2018 to May 2019. These data were obtained through MedLine, PubMed, Scielo, LILACS and BBO databases. Results: The results showed that photodynamic therapy associated with laser is effective as an adjunct to conventional endodontic treatment, being one of the main uses to assist in the disinfection of root canals, which consists of using a light source with a specific wavelength capable of stimulating a photosensitive agent, which results in oxidative damage, causing cell death. Studies of its performance in cavity preparation, control of pulp vitality, pulp capping and pulpotomy, cleaning of root ducts, obturation and adhesion of endodontic cements, retreatment, periapical surgeries, pain reduction and postoperative repair. Conclusion: In conclusion the photodynamic therapy used as a coadjuvant to conventional endodontic therapy increases the predictability of treatment success since the knowledge about the fundamentals and interactions with biological tissues are clarified and dominated by the professional.

Descriptors: Endodontics; Lasers; Photochemotherapy; Laser Therapy.

\section{Resumen}

Introducción: La terapia fotodinámica asociada con el láser se ha estudiado y aplicado en varias especialidades de odontología con el objetivo de utilizar técnicas menos invasivas. Objetivo: Este estudio tiene como objetivo presentar a los dentistas y a la sociedad la nueva cara de la Odontología centrada en el uso de la terapia fotodinámica asociada con el láser en la práctica de endodoncia, así como presentar los aspectos generales de la terapia con láser. Material y método: Es una revisión de literatura, caracterizada como descriptiva y exploratoria. Se realizaron búsquedas en libros, artículos científicos, monografías, disertaciones y tesis, desde agosto de 2018 hasta mayo de 2019. Estos datos se obtuvieron a través de las bases de datos MedLine, PubMed, Scielo, LILACS y BBO. Resultados: Los resultados mostraron que la terapia fotodinámica asociada con el láser es efectiva como complemento del tratamiento endodóntico convencional, siendo uno de los principales usos para ayudar en la desinfección de los conductos radiculares, que consiste en utilizar una fuente de luz con una longitud de onda específica capaz de estimular un agente fotosensible, que produce daño oxidativo, causando la muerte celular. También se observan estudios de su desempeño en la preparación de cavidades, control de la vitalidad pulpar, recubrimiento pulpar y pulpotomía, limpieza del conducto radicular, llenado y adhesión de cementos endodónticos, retratamiento, cirugías periapicales, reducción del dolor y reparación postoperatoria. Conclusión: Se concluyó que la terapia fotodinámica utilizada como complemento de la terapia endodóntica convencional aumenta la previsibilidad del éxito del tratamiento, siempre que el profesional aclare y domine el conocimiento sobre los fundamentos y las interacciones con los tejidos biológicos.

Descriptores: Endodoncia; Láseres; Fotoquimioterapia; Terapia por Láser.

INTRODUÇÃO

$$
\text { O tratamento endodôntico convencional }
$$

tem como finalidade devolver aos canais radiculares às condições ideais de antissepsia, entretanto, a remoção completa da microbiota ainda é um desafio devido à complexa anatomia dos canais radiculares. Portanto, novas técnicas e novos métodos estão sendo utilizados com objetivo de minimizar as taxas de insucesso. Com a finalidade de melhorar o tratamento convencional, a terapia fotodinâmica associada ao laser apresenta-se como alternativa coadjuvante na eliminação dos microrganismos ${ }^{1,2}$.

A Terapia Fotodinâmica (PhotoDynamic 
Therapy - PDT) caracteriza-se pela utilização da luz (laser ou LED), associado a fotossensibilizadores. Deste modo, a desinfecção dos canais radiculares através desta terapia consiste em utilizar uma fonte de luz com comprimento de onda específico capaz de estimular um agente fotossensível (corante), que na presença de oxigênio resulta em danos oxidativos, causando a morte celular ${ }^{3,4}$.

Essa terapia tem sido estudada e aplicada em diversas especialidades da Odontologia, objetivando o uso de técnicas menos invasivas, como em preparo cavitário de tratamentos restauradores, em periodontia, na bioestimulação de tecidos moles e na redução dos microrganismos na Endodontia ${ }^{5}$.

Nesse contexto, a terapia fotodinâmica desponta como uma nova terapia, coadjuvante ao tratamento endodôntico convencional, na tentativa de eliminar microrganismos persistentes ao preparo químico-mecânico. Sendo uma técnica de fácil e rápida aplicação clínica e indolor, não desenvolve resistência microbiana, podendo ser indicada em tratamentos endodônticos em sessão única ou em múltiplas sessões ${ }^{6,7}$.

Diante do exposto, o presente estudo tem como objetivo geral apresentar aos cirurgiõesdentistas e a sociedade a nova face da Odontologia voltada à utilização da terapia fotodinâmica associada a laser na prática endodôntica. De forma mais específica, apresentar os aspectos gerais da laserterapia e o cenário atual do seu uso na Endodontia.

\section{MATERIAL E MÉTODO}

O presente trabalho trata-se de uma pesquisa descritiva e exploratória que é um tipo específico de produção científica, baseada em levantamento de textos, como livros, artigos científicos, dicionários, jornais, revistas, entre outros. Vale ressaltar que os artigos científicos constituem o primeiro foco dos pesquisadores, porque é neles que se pode encontrar conhecimento científico atualizado. A pesquisa da literatura compreende oito fases distintas, são elas: Escolha do tema, elaboração do plano de trabalho, identificação, localização, compilação, fichamento, análise e interpretação, redação ${ }^{8}$.

Também chamada de pesquisa de fontes secundárias, a pesquisa bibliográfica trata-se de um levantamento de referências já publicadas, em forma de artigos científicos, livros, teses de doutorado, dissertações de mestrado ${ }^{8}$. Foram utilizados livros de acordo com a área temática e artigos científicos acessados nas bases de dados BVS, PubMed,
Scielo e MEDLINE, LILACS e BBO, sem restrição do período de publicação. Foram selecionados artigos científicos na língua portuguesa e inglesa.

Os descritores utilizados foram "Lasers", "Endodontia", Fotoquimioterapia" e "Terapia a Laser". O período de realização das buscas dos materiais foi do mês de agosto de 2018 a maio de 2019.

Nesta etapa foi realizada a leitura dos materiais obtidos, primeiramente de forma rápida para selecionar a obra e analisar se ela é de interesse da pesquisa. Depois de selecionadas as obras, foram lidas de forma mais aprofundada a fim de responder 0 problema da pesquisa e os objetivos.

RESULTADOS E DISCUSSÃO

Os limites atuais das estratégias de desinfecção endodôntica não são apenas decorrentes do modo de crescimento do biofilme bacteriano dentro dos canais radiculares, são igualmente decorrentes da complexidade anatômica do sistema de canais radiculares, de sua estrutura, da composição dentinária e dos fatores associados com desinfetantes químicos. Grande parte da anatomia endodôntica permanece não instrumentada após o tratamento convencional e até mesmo as partes acessíveis dos canais radiculares são cobertas por smear layer, o que resulta como um subproduto da instrumentação e atua como uma barreira para irrigantes, medicamentos e até mesmo influenciando a qualidade do preenchimento endodôntico. Dessa forma, estratégias de desinfecção avançada em endodontia são continuamente desenvolvidas e testadas para enfrentar esses desafios e proporcionar o máximo conforto e bem-estar aos pacientes ${ }^{9}$.

Segundo Silva et al. $^{10}$, a redução microbiana nos canais radiculares teve maior efetividade quando houve associação da instrumentação com a PDT, comparado ao tratamento que se utilizou apenas a instrumentação. Além disso, não promove resistência microbiana, fator relevante que provoca insucesso das técnicas convencionais. Silva et al. ${ }^{10}$ alertam ainda que a PDT apresenta diferença de efetividade quando os microrganismos estão dispostos isoladamente e em forma de biofilme. Os autores acrescentam que existe efetividade da PDT frente ao Enterococcus faecalis, espécie mais resistente ao tratamento endodôntico convencional ${ }^{10,11}$, porém é necessário maior aprimoramento da técnica utilizada.

Os autores relatam que quando estes 
microrganismos estão dispostos em forma isolada a terapia mostra melhores resultados comparado aos microrganismos dispostos em biofilme, corroborando com o estudo de Silva et al. $^{10}$. Além disso, reafirmam que a complexidade anatômica do sistema de canais radiculares inviabiliza a total eliminação de microrganismos; entretanto, a utilização de corantes, que funcionam como agente fotossensível sensibilizando os microrganismos por meio de luz com comprimento de onda apropriado, causa efeito bactericida ${ }^{6}$.

$\mathrm{Na}$ Endodontia, os fotossensibilizadores derivados de fenotiazinas têm sido amplamente utilizados, embora haja risco de manchamento dental. Vários estudos apontam que os corantes azuis são potentes sensibilizadores para uma determinada faixa de bactérias quando irradiados por lasers que emitem no espectro do vermelho visível. Deste modo, o azul de ortotoluidina e o azul de metileno, associados a um laser de comprimento de onda por volta de $630 \mathrm{~nm}$, apresentam melhor resultado na eliminação de bactérias e fungos. Os mesmos foram testados para verificar qual deles teria a ação bactericida mais eficaz contra os diferentes tipos de bactérias Gram positivas e negativas. Os resultados demonstraram que ambos foram eficientes usando laser de emissão vermelha, porém, o azul de ortotoluidina apresentou melhor efeito bactericida que o azul de metileno ${ }^{12}$. Todavia, Fimple et al. ${ }^{12}$ afirmam que na PDT tem-se usado 0 azul de metileno como fotossensibilizador devido ao seu sucesso contra bactérias gram-positivas e gramnegativas, além da capacidade de penetrar nos túbulos dentinários.

Olivi et al. ${ }^{13}$ ressaltam que uma das principais vantagens da utilização dos lasers é a desinfecção por estes conseguida, bem como a melhor limpeza e remoção de smear layer, permitindo que as paredes dentinárias tornemse lisas e uniformes em menor tempo quando comparado apenas a instrumentação convencional. Os autores asseguram que a morfologia produzida após irrigação com $\mathrm{NaOCl}$ e clorexidina é de túbulos fechados e presença de smear layer, deste modo, obteve-se melhores resultados ao nível das paredes quando se utilizou o laser juntamente com EDTA, possibilitando superfícies limpas e sem vestígios de danos térmicos ${ }^{14-16}$.

Além da diminuição do número de microrganismos e redução da smear layer, permitindo paredes dentinárias lisas $e$ uniformes, vários autores apresentam diversos outros benefícios da PDT como: técnica de fácil aplicação, permite tratamento em sessão única e indolor, facilita a desobturação e a adesão dos cimentos endodônticos, favorece o prognóstico dos tratamentos endodônticos cirúrgicos, promove a diminuição da sensibilidade pósoperatória, controle da hemostasia, e não apresenta efeitos colaterais ${ }^{1,6,17-20}$.

Reis ${ }^{4}$ enumera e ressalta ainda algumas outras vantagens quanto ao emprego da luz laser em Endodontia, tais como: rápida ação bactericida em biofilmes, fácil penetração do fotossensibilizador nos túbulos dentinários, redução da permeabilidade e micro infiltração apical limitando a toxicidade aos tecidos de sustentação, ausência de efeitos térmicos ao periodonto, em dentes vitais apresenta remoção e coagulação do tecido pulpar e de resíduos em terço apical do canal radicular e vaporização de resíduos, substâncias orgânicas e dentina infectada em dentes sem vitalidade pulpar.

Ainda que os estudos relatados verifiquem os benefícios da utilização dos lasers, há ainda um fator que merece um cuidado especial que é o aumento da temperatura sobre a estrutura dentinária. Dessa forma, os parâmetros de energia empregados devem ser seguros para evitar danos térmicos irreversíveis à dentina, ao cemento e às fibras do ligamento periodontal ${ }^{21}$. Olivi et al. $^{13}$ reafirmam que a utilização dos lasers para a preparação dos canais pode aumentar a temperatura, possibilitando a ocorrência de danos irreversíveis nas paredes dentinárias, e esses efeitos morfológicos do laser nas paredes radiculares têm vindo a ser descritos como efeitos colaterais da limpeza e descontaminação dos canais com diferentes tipos de lasers.

Pécora et al. ${ }^{22}$ verificaram o aumento da temperatura na superfície externa das raízes dos dentes após o uso do laser Er:YAG em canais radiculares previamente instrumentados sem irrigação e com água como irrigante. Os resultados obtidos mostraram que quando a água era utilizada como irrigante ao mesmo tempo em que se aplicava o laser, os aumentos de temperatura foram inferiores quando não se utilizou solução irrigante. Assim, os autores indicam boa refrigeração e irrigação durante o uso do laser no interior dos canais radiculares, para se evitar o aquecimento da estrutura dentinária ${ }^{21}$. Os resultados corroboram com os de Kimura et al. $^{23}$, que observaram que a presença de água é fundamental para evitar lesões térmicas, especialmente nos lasers de érbio, pois a presença da água nas soluções irrigadoras limita a interação térmica entre o laser e a parede dentinária.

Ackroyd et al. ${ }^{24}$ afirmam que lasers de 
baixa potência não produzem efeito térmico, porque a variação de temperatura é de aproximadamente $0,5^{\circ} \mathrm{C}$. Tampouco promovem alterações morfológicas na estrutura dentária, além de serem mais portáteis, de mais fácil manuseio e ter custo reduzido em relação aos lasers de alta potência. Como a dose da radiação é facilmente calculada, a área a ser tratada pode ser bem delimitada e a radiação pode ser transmitida por fibras ópticas, que podem receber adaptação para atingir o alvo.

No estudo de Wang et al. ${ }^{25}$ constatou-se que uma das principais preocupações na utilização do laser é o sobreaquecimento do osso alveolar e tecidos circundantes. A temperatura mínima a qual o osso demonstra sensibilidade ao calor é cerca de $47^{\circ} \mathrm{C}$, o que representa aproximadamente um aumento de $10^{\circ} \mathrm{C}$ por minuto. Acima dos $53^{\circ}$ ocorre necrose óssea. Neste estudo, o aumento de temperatura máximo atingido com a utilização do laser díodo foi de $8,1^{\circ} \mathrm{C}$, abaixo do limite que causa danos aos tecidos.

Namour et al. $^{26}$ realizaram um estudo com o objetivo de determinar a faixa de segurança dos parâmetros de irradiação durante a aplicação endodôntica do laser de $\mathrm{Nd}$ :YAP que pode ser usado sem danificar e superaquecer 0 tecido periodontal. Vinte e sete dentes humanos extraídos com raízes simples, livres de cáries foram utilizados. As coroas foram seccionadas para obter um comprimento de canal radicular de $11 \mathrm{~mm}$. Os aumentos de temperatura na superfície das raízes foram medidos por um termopar durante a irradiação com laser $\mathrm{Nd}$ :YAP dos canais radiculares em diferentes densidades de energia. Cada irradiação foi realizada em um canal irrigado continuamente com solução de $\mathrm{NaOCl} 2,25 \%$. As médias e desvios padrão dos aumentos de temperatura nas superfícies radiculares foram inferiores a $10^{\circ} \mathrm{C}$ (nível do limiar seguro). Dentro das limitações deste estudo e sob condições específicas de irradiação, o feixe de laser $\mathrm{Nd}$ :YAP pode ser considerado inofensivo para tecidos periodontais durante aplicações endodônticas, corroborando com estudos de Reis ${ }^{4}$.

No que diz respeito a desvantagens da utilização da PDT na terapia endodôntica, Mathew e Thangaraj ${ }^{17}$ destacam a necessidade de ajustar os comprimentos de onda conforme os tratamentos efetuados e a inexistência de protocolos universalmente aceitos. Além disso, acrescenta-se como inconveniente na utilização dos lasers o preço elevado do aparelho e a necessidade da habilitação do operador para a sua correta utilização, que requer geralmente algum tempo de aprendizagem ${ }^{5,27}$

Sendo assim, apesar da atuação do laser na endodontia permitir diversos benefícios, é necessário que as técnicas, efeitos e mecanismo de ação seja conhecido por profissionais que fazem o uso desta terapia ${ }^{1,5}$.

Diante do exposto, podemos observar que $o$ uso dos lasers vem sendo amplamente estudado e indicado na terapia endodôntica, já que a técnica possui numerosas vantagens e benefícios. No entanto, como toda técnica, é fundamental que se conheça bem os seus princípios básicos, principalmente pelo fato dos seus efeitos e mecanismo de ação serem bastante complexos. Desta forma, os fundamentos e a interação dessa luz com os tecidos biológicos são conhecimentos que devem ser esclarecidos e dominados pelo profissional cirurgião-dentista ${ }^{5,27}$.

CONCLUSÃO

De acordo com a literatura estudada concluiu-se que a Terapia Fotodinâmica associada a laser como coadjuvante ao tratamento endodôntico convencional apresenta diversas utilizações comprovadas, sendo uma das principais utilizações auxiliar na desinfecção dos canais radiculares. Assim sendo, pode aperfeiçoar a terapia, aumentando assim a previsibilidade de sucesso. Entretanto, deve-se levar em consideração que o conhecimento sobre os fundamentos e interações com os tecidos biológicos devem ser esclarecidos e dominados pelo profissional; e são necessários mais estudos e ajustes a fim de padronizar algumas características da técnica.

\section{REFERÊNCIAS}

1. Ferreira CLR, Anacleto FN, Santos CHSD, Vieira AD, Vance R. Uso de ultrassom e laser na descontaminação do sistema de canais radiculares. Dent press endod. 2017;7(3):27-33.

2. Lacerda MFLS, Lima CO, Lacerda GP, Campos $\mathrm{CN}$. Evaluation of the dentin changes in teeth subjected to endodontic treatment and photodynamic therapy. Rev Odontol UNESP. 2016;46(6):339-43.

3. Konopka K, Goslinski T. Photodynamic Therapy in Dentistry. J Dent Res. 2007;86(8):694-707.

4. Reis F. Tecnologias endodônticas. São Paulo: Santos; 2015.

5. Cavalcanti TM, Almeida-Barros RQ, Catão MHCV, Feitosa APA, Lins RDAU. Conhecimento das propriedades físicas e da interação do laser com os tecidos biológicos na odontologia. An Bras Dermatol. 2011; 86(5):955-60.

6. Paiva PCP, Nunes E, Silveira FS, Cortes MIS. Aplicação clínica do laser em endodontia. RFO. 2007;12(2):84-8. 
7. Chen Y, Chen XL, Zou XL, Chen SZ, Zou J, Wang Y. Efficacy of low-level therapy in pain management after root canal treatment of retreatment: a systematic review. Lasers Med Sci. 2019;34(7):1305-16.

8. Lakatos EM, Marconi MA. Fundamentos de metodologia científica. 8. ed. São Paulo: Atlas, 2017.

9. Todea DCM, Luca RE, Balabuc CA, Miron MI, Locovei C, Mocuta DE. Scanning electron microscopy evaluation of the root canal morphology after Er:YAG laserirradiation. Rom J Morphol Embryol. 2017:59(1):269-75.

10. Silva FC, Freitas LRP, Lourenço APA, Braga Junior ACR, Jorge AOC, Oliveira LD et al. Análise da efetividade da instrumentação associada à terapia fotodinâmica antimicrobinana e a medicação intracanal na eliminação de biofilmes de Enterococcus faecalis. Braz Dent Sci. 2010;13(5):31-8.

11. Asnaashari $M$, Homayuni $H$, Paymanpour $P$. The antibacterial effect of additional photodynamic therapy in failed endodontically treated teeth: a pilot study. J Lasers Med Sci. 2016;7(4):238-42.

12. Fimple JL, Fontana CR, Foschi F, Ruggiero K, Song $X$, Pagonis TC et al. Photodynamic treatment of endodontic polymicrobial infection in vitro. J Endod. 2008;34(6):728-34.

13. Olivi G, Crippa R, laria G, Kaitsas V, Divito E, Benedicenti S. Laser in Endodontics (Part I). Roots. 2011;7(1):6-9.

14. Ashraf H, Asnaashari M, Darmiani S, Birang R. Smear layer removal in the apical third of root canals by two chelating agents and laser: a comparative in vitro study. Iran Endod J. 2014; 9(3):210-14.

15. Li Z, Fang C. [Bactericidal effects and smear layer removal of Er:YAG-laser radiation against Enterococcus faecalis in root canals]. Zhong Nan Da Xue Xue Bao Yi Xue Ban. 2017;42(3):328-33.

16. Moura-Netto C, Mello-Moura AC, Palo RM, Prokopowitsch I, Pameijer $\mathrm{CH}$, Marques MM. Adaptation and penetration of resin-based root canal sealers in root canals irradiated with highintensity lasers. J Biomed Opt. 2015;20(3):038002.

17. Mathew S, Thangaraj DN. Laser in Endodontics. J Indian Acad Dent Spec. 2010;1(1)31-7.

18. Tachinami $\mathrm{H}$, Katsumi I. Removal of root filling materials using Er: YAG laser irradiation. Dent Mater J. 2010;29(3):246-52.

19. Asnaashari M, Ashraf $H$, Daghayeghi $A H$, Mojahedi SM, Marhabi SA. Management of post endodontic retreatment pain with low level laser therapy. J Lasers Med Sci.2017;8(3):128-31.
20. Nabi S, Amin K, Masoodi A, Farooq R, Purra AR, Ahangar FA. Effect of preoperative ibuprofen in controlling postendodontic pain with and without low-level laser therapy in single visit endodontics: A randomized clinical study. Indian J Dent Res. 2018;29(1):46-50.

21. Melo TAF, Oliveira EPM, Barletta FB, Becker AN, Kunert GG. Aplicação do laser na terapia endodôntica. Stomatos, 2007;28(24):53-61.

22. Pécora JD, Cussioli AL, Guerisoli DMZ, Marchesan MA, Sousa-Neto MD, BrugneraJunior A. Evaluation of Er:YAG Laser and EDTAC on Dentin Adhesion of Six Endodontic Sealers. Braz Dent J. 2001;12(1):27-30.

23. Kimura $Y$, Wilder-smith $P$, Matsumoto $K$. Lasers in endodontics: a review. Internetional Endod J. 2001;33(3):173-85.

24. Ackroyd R, Kelty C, Brown N, Reed M. The history of photodetection and photodynamic therapy. Photochem Photobiol. 2001;74(5): 656-69.

25. Wang QQ, Zhang CF, Yin XZ. Evaluation of the bactericidal effect of $\mathrm{Er}, \mathrm{Cr}$ :YSGG, and $\mathrm{Nd}$ : YAG lasers in experimentally infected root canals. J Endod. 2007;33(7):830-32.

26. Namour A, Geerts S, Zeinoun T, De Moor R, Nammour S. Safety irradiation parameters of nd:yap laser beam for endodontic treatments: an in vitro study. Biomed Res Int, 2016. ;2016:4741516.

27. Siqueira MBLD, Lúcio PSC, Godoy GP, Vasconcelos-Catão MHC. A terapia com laser em especialidades odontológicas. Rev Cuba Estomatol. 2015;52(2):143-49.

\section{CONFLITO DE INTERESSES}

Os autores declaram não haver conflitos de interesse

\section{AUTOR PARA CORRESPONDÊNCIA}

\section{Rachel Reinaldo Arnaud}

Rodovia BR-230, km 22, s/n - Água Fria

58053-000 João Pessoa - PB, Brasil

E-mail: rachel.arnaud@unipe.edu.br
Submetido em 28/05/2020 Aceito em 17/01/2021 